\title{
An Exploration about the Interaction of Mild Steel with Hydrochloric Acid in the Presence of N-(Benzo[d] Thiazole-2-yl)-1-Phenylethan-1-Imines
}

\author{
Bhawna Chugha, ${ }^{\dagger}$, Ashish Kumar Singh ${ }^{b,}{ }^{*}, \dagger$, Sanjeeve Thakura, Balaram Panic, Ajit Kumar \\ Pandey $^{a}$, Hassane Lgaz, *, Ill-Min Chungd, *, Eno E. Ebenso ${ }^{\mathrm{e}}$ \\ a Department of Chemistry, Netaji Subhas Institute of Technology, New Delhi-110078, India \\ bepartment of Applied Science, Bharati Vidyapeeth College of Engineering, New Delhi-110063, India \\ 'Department of Chemistry, Bhaskaracharya College of Applied Science, University of Delhi, New \\ Delhi-110078, India \\ ${ }^{\mathrm{d} D e p a r t m e n t}$ of Crop Science, College of Sanghur Life Science, Konkuk University, Seoul 05029, South \\ Korea \\ 'Material Science Innovation \& Modelling (MaSIM) Research Focus Area, Faculty of Natural and \\ Agricultural Sciences, North-West University, Private Bag X2046, Mmabatho 2735, South Africa \\ $\dagger^{\dagger}$ First author \\ *Corresponding author \\ Email: ashish.singh.rs.apc@itbhu.ac.in \\ hlgaz@konkuk.ac.kr \\ imcim@konkuk.ac.kr \\ Tel: +91-9560285447
}




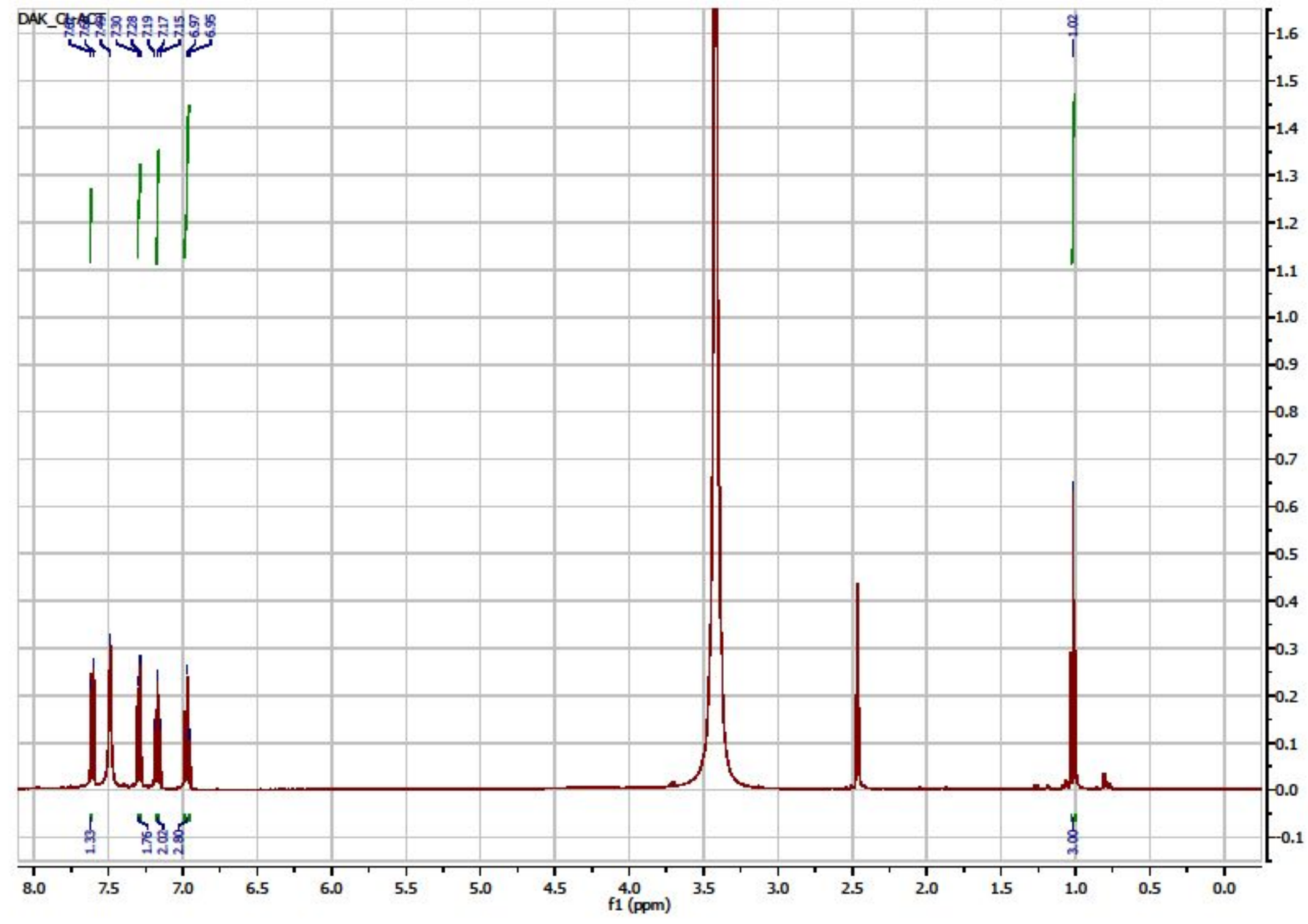

Fig S1 ${ }^{1} \mathrm{H}$ NMR spectrum of BTCPEI 


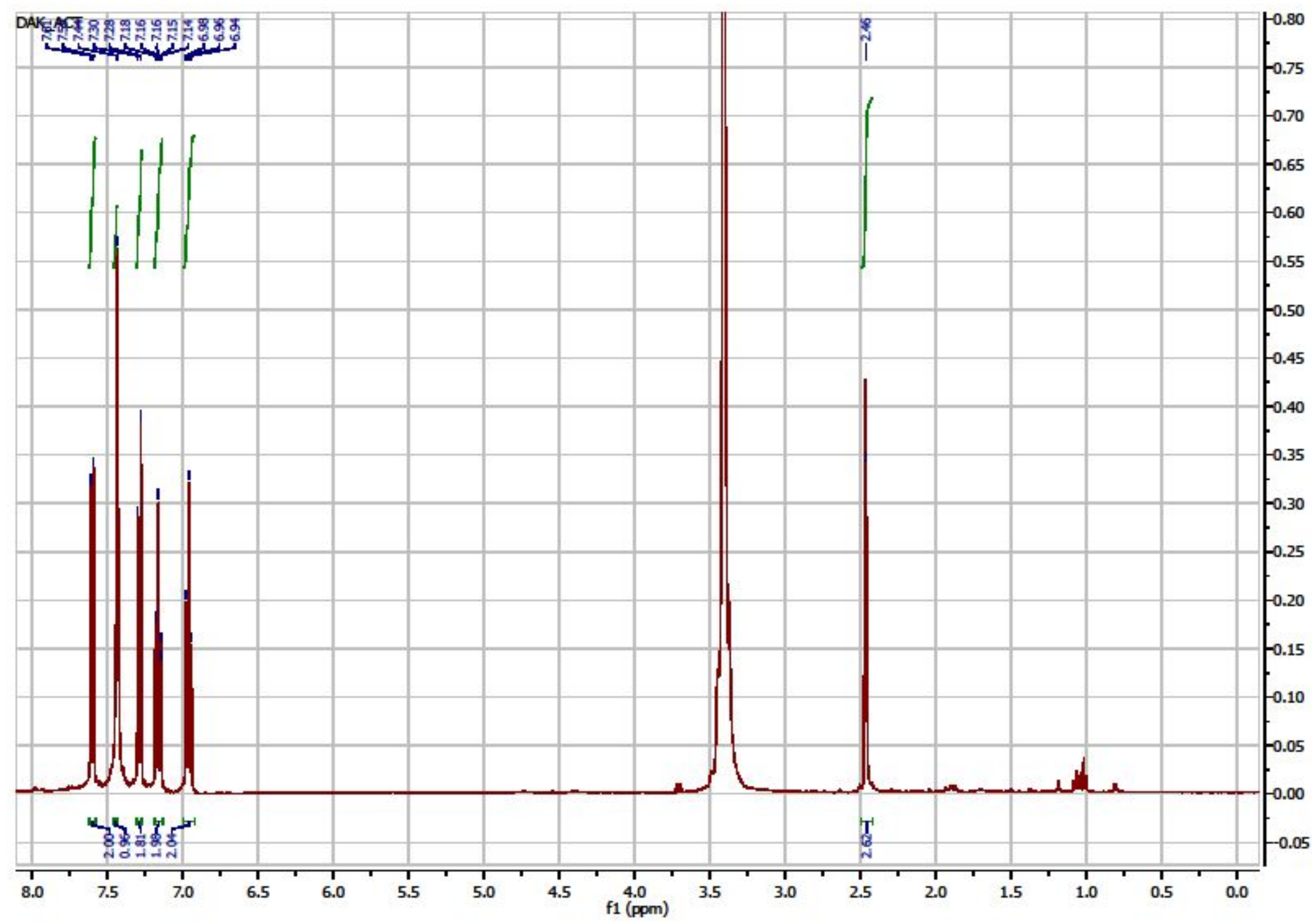

Fig S2 ${ }^{1} \mathrm{H}$ NMR spectrum of BTPEI 


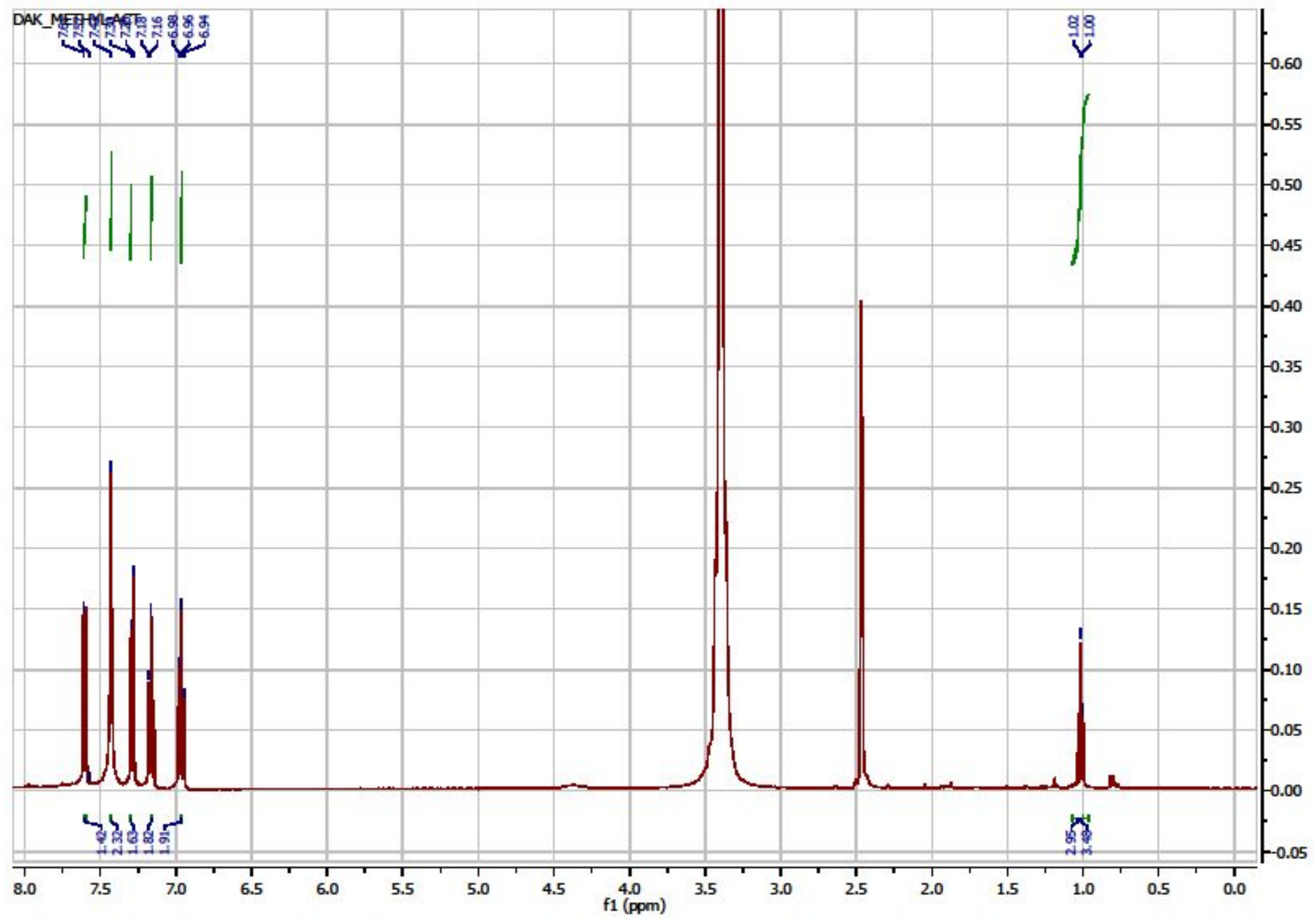

Fig S3 ${ }^{1} \mathrm{H}$ NMR spectrum of BTTEI 


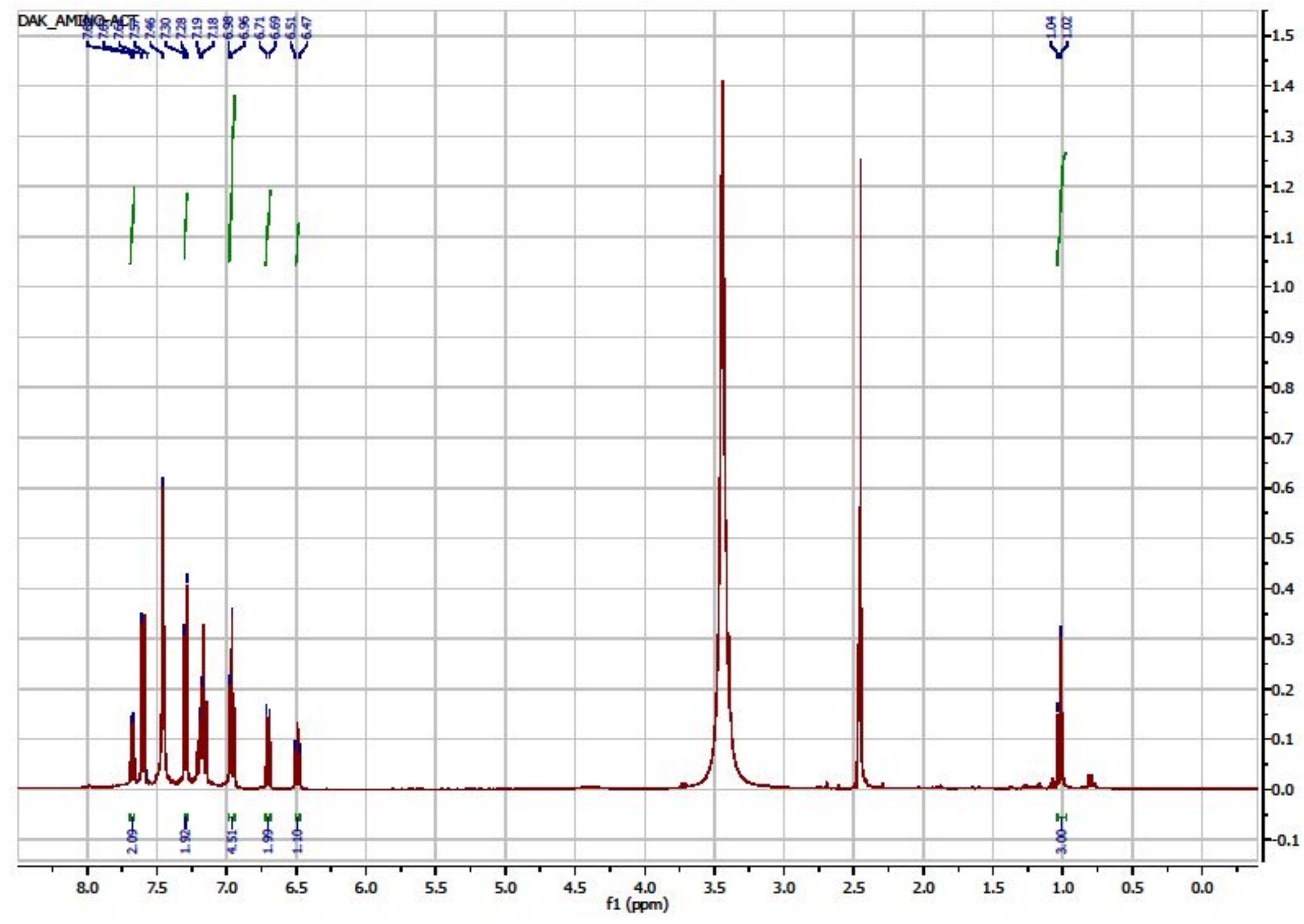

Fig S4 ${ }^{1} \mathrm{H}$ NMR spectrum of BTPIA 

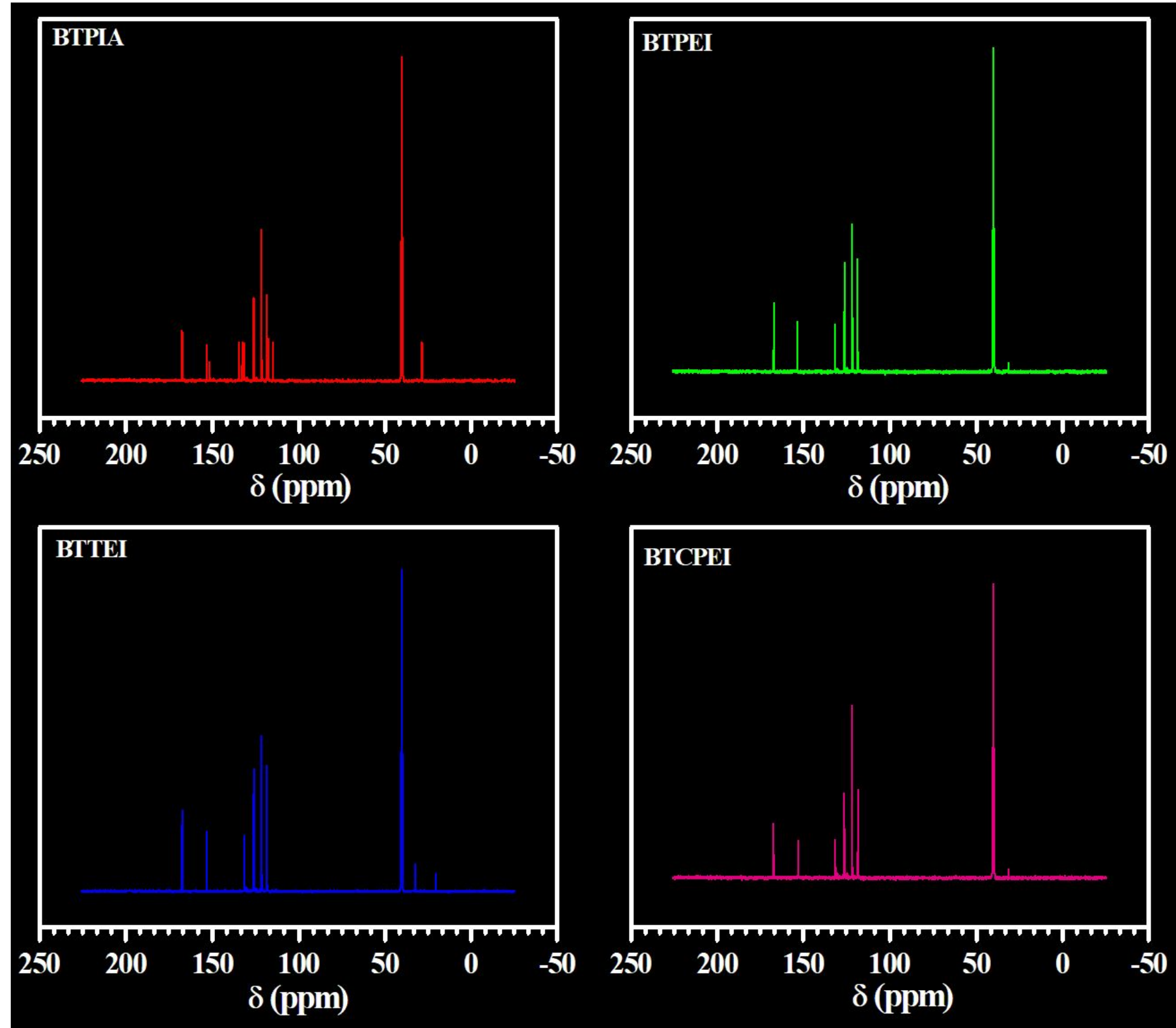

Fig S5 13C NMR spectra of BTCPEI, BTTEI, BTPEI and BTPIA 


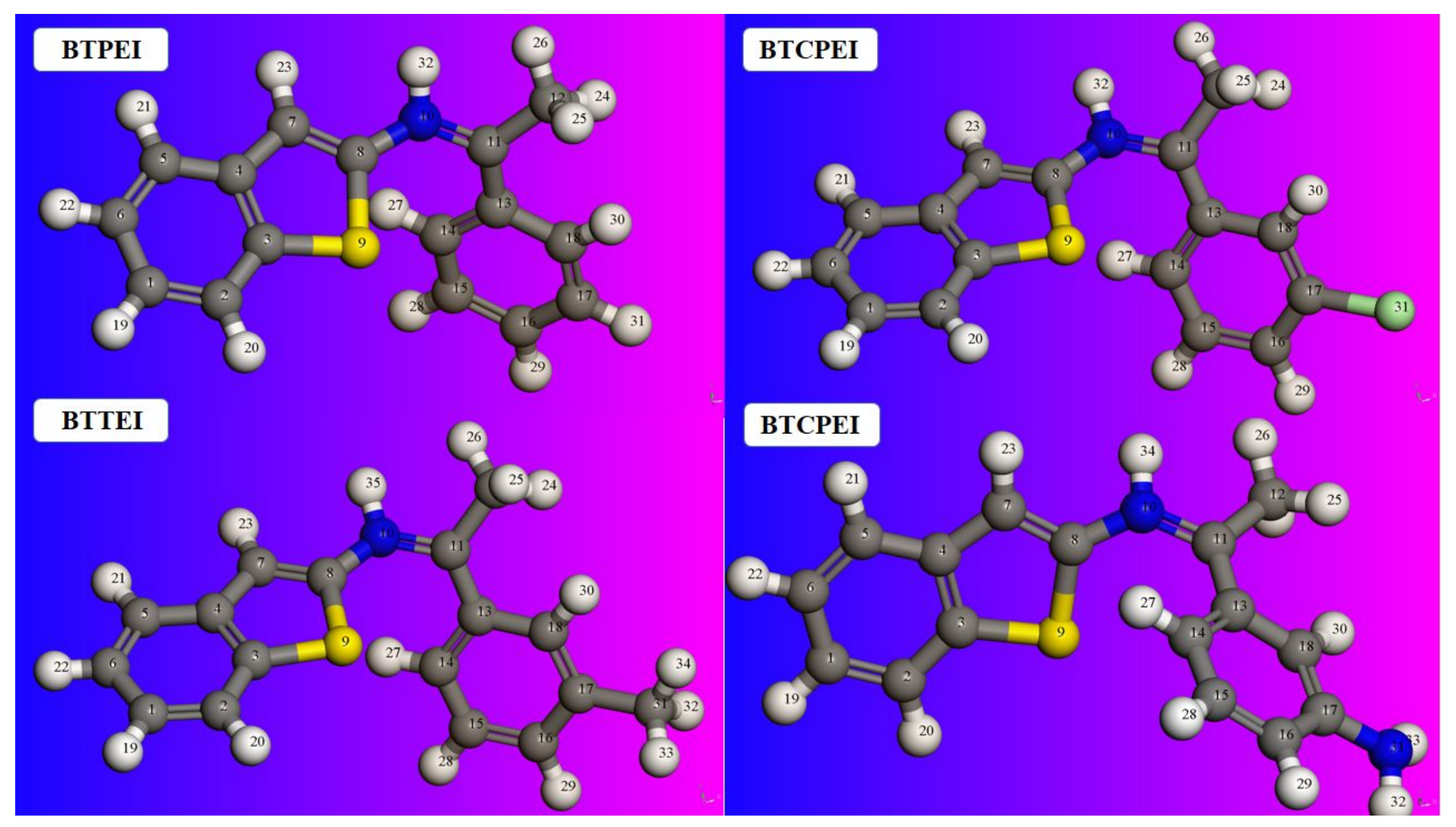

Fig S6 Optimized molecular structure of protonated form of investigated compounds obtained from DFT calculations 


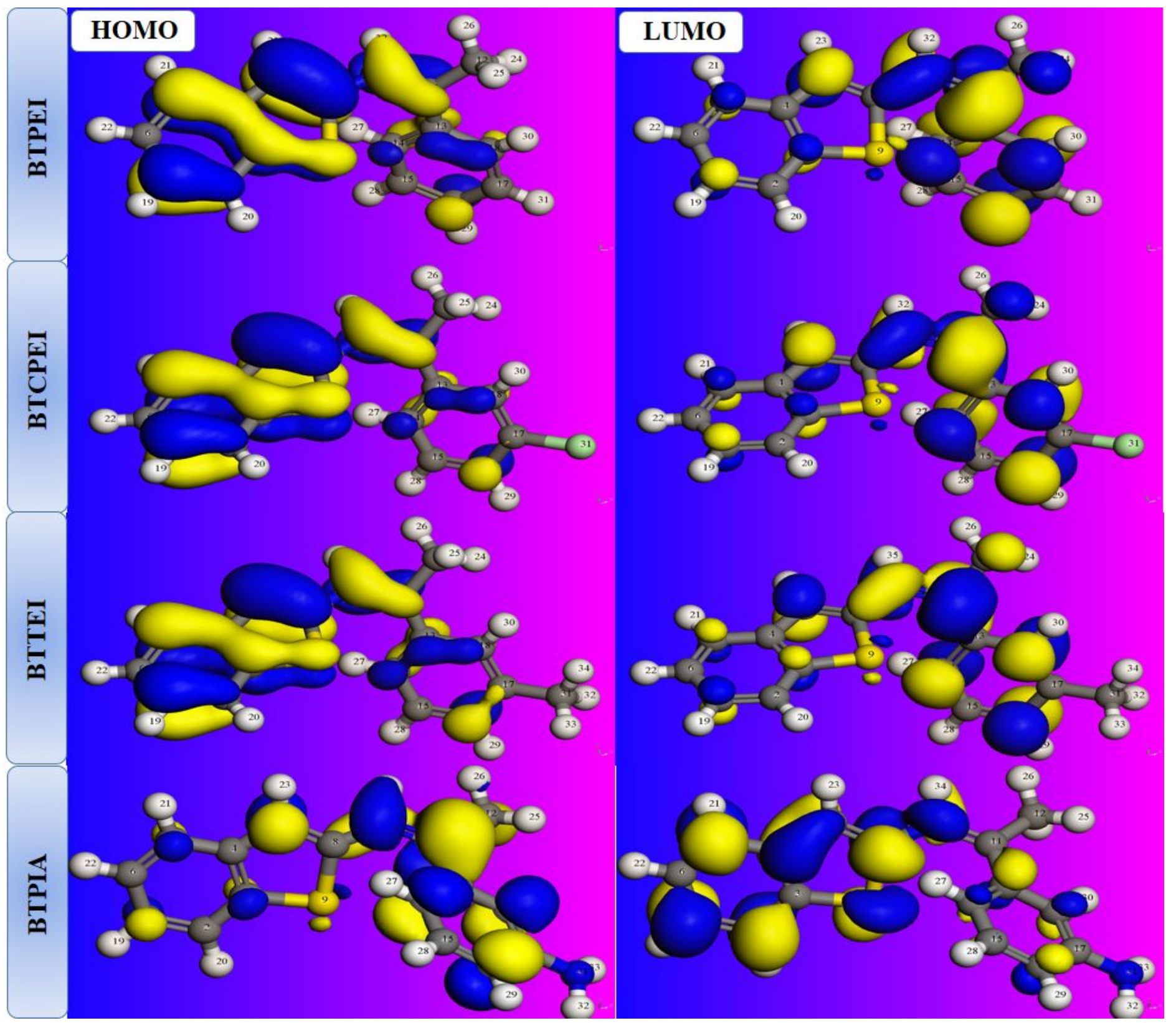

Fig S7 Frontier molecule orbital density distributions for protonated form of investigated compounds obtained from DFT calculations 